\title{
Attempt to Control Sequence of Branched Polysaccharide with Enzymatic Hydrolysis and/or Copolymerization
}

\author{
Kenichi Hatanaka, ${ }^{\dagger}$ Soo-Chang Song, Atsushi Maruyama, \\ Akira Kobayashi, ${ }^{*}$ Hiroyoshi Kuzuhara, ${ }^{* *}$ \\ and Toshihiro AKAIKE \\ Department of Biomolecular Engineering, Tokyo Institute of Technology, \\ Nagatsuta, Midori-ku, Yokohama 227, Japan \\ * KAST (Kanagawa Academy of Science and Technology), \\ Sakado, Takatsu-ku, Kawasaki 213, Japan \\ ** RIKEN (The Institute of Physical and Chemical Research), \\ Hirosawa, Wako-shi, Saitama 351, Japan
}

(Received August 14, 1992)

\begin{abstract}
O$-benzyl-( $1 \rightarrow 6)$ - $\alpha$-D-glucopyranan, which was synthesized by ringopening polymerization of 1,6-anhydro-3-O-benzoyl-2,4-di- $O$-benzyl- $\beta$-D-glucopyranose $\left(\mathrm{M}_{1}\right)$ and subsequent selective deprotection of the obtained polymer, ${ }^{1}$ was glucosylated to give branched polysaccharide having several different anomeric ratios of branching units. $\beta$-Glucosidase (cellulase) cleaved only $\beta$-glucosidic linkage of the synthesized polysaccharide to give a $\alpha$-glucose-branched polysaccharide with controlled branching distribution. The sequencial control of the hydroxyl group (branching point) in the polysachharide was attempted also by copolymerization. $\mathrm{M}_{1}$ was found to copolymerize with tri-O-benzyl-levoglucosan $\left(\mathrm{M}_{2}\right)$ with phosphorus pentafluoride as catalyst in methylene chloride at $-60^{\circ} \mathrm{C}$, giving highly stereoregular copolymers. The monomer reactivity ratios calculated by Kelen-Tüdös method were $r_{1}=0.27$ and $r_{2}=2.5$.
\end{abstract}

KEY WORDS Branched Polysaccharide / Sequencial Control / Ring-

Opening Polymerization / $\beta$-Glucosidase / Enzymatic Hydrolysis /

Glycosylation / Hypoglycaemic Activity / Bioactive Polysaccharide / Cellulase

Many kinds of oligosaccharide chain portion in glycoconjugates (glycoprotein, glycolipid, etc.) have been precisely synthesized according to the technology of glycosynthesis with the view of elucidating the mechanisms of bioactivities of saccharide chains and preparing the biomimetic materials. ${ }^{2-4}$ The relationship between the chemical structure of bioactive polysaccharides and the biological activity has been also reported in detail. Branching sugar units are sometimes important for the polysaccharides to exhibit biological activities. Panaxan A, which is a natural branched polysaccharide isolated from ginseng, shows biological activity of lowering the blood

\footnotetext{
† To whom correspondence should be addressed.
}

glucose level. ${ }^{5}$ The reported chemical structure of Panaxan A was $(1 \rightarrow 6)$ - $\alpha$-D-glucopyranan having $(1 \rightarrow 3)-\alpha$-D-glucopyranosyl branch. ${ }^{6}$

The cationic ring-opening polymerization of 1,6-anhydrosugar derivatives gave stereoregular $(1 \rightarrow 6)-\alpha$-linked polysaccharides. ${ }^{7-9}$ For the purpose of obtaining branched polysaccharides, polymerizations of 1,6-anhydro-disaccharide derivatives have been attempted. However, most of 1,6-anhydro-disaccharide derivatives are not suitable for the synthesis of branched polysaccharides because of their low polymerizability. ${ }^{10,11}$ Only 1,6anhydromannose derivative, which is the most reactive monomer among 1,6-anhydrosugars, 
gave stereoregular branched polysaccharide. ${ }^{12}$ Synthesis of the branched polysaccharide was attempted also by the regiospecific glycosylation of the partially protected polysaccharide, which was prepared by ring-opening polymerization of 1,6-anhydrosugar with two kinds of protective groups and subsequent selective deprotection of the polymer. ${ }^{13-15}$ However, there is no report about the control of the branching distribution. And copolymerization was not attempted for the purpose of controlling the branched sequence.

In this investigation, the control of the branching distribution in the synthesis of branched polysaccharide with enzymatic hydrolysis is reported. And the possible control of the branched sequence in copolymerization is also examined.

\section{EXPERIMENTAL}

\section{General Methods}

NMR spectra were recorded on a JEOL EX-270 spectrometer in $\mathrm{CDCl}_{3}$ and $\mathrm{D}_{2} \mathrm{O}$ using TMS and DSS as internal standard, respectively. GPC was carried out on $1 \%$ solutions of the polymer in tetrahydrofuran with a Shimadzu liquid chromatograph (model LC9A, columns: GPC-803 and GPC-804). The number-average molecular weights calculated by GPC were based on polystyrene calibration curve. The number-average molecular weights of water-soluble polysaccharides were determined by aqueous phase GPC (columns: Asahipak GS-510 (Asahi Chemical Industry)) using standard pullulan as reference. Optical rotations were recorded with a JASCO Model 370 polarimeter at room temperature in chloroform or water using a $0.5-\mathrm{dm}$ cell.

\section{Monomers}

1,6-Anhydro-3- $O$-benzoyl-2,4-di- $O$-benzyl$\beta$-D-glucopyranose ${ }^{1}(1)$ and 1,6-anhydro-2,3,4tri- $O$-benzyl- $\beta$-D-glucopyranose ${ }^{7,8}$ (2) were synthesized from levoglucosan according to the literature.

\section{Polymerization and Debenzoylation}

Polymerization of $\mathbf{1}$, copolymerization of 1 with 2 , and regioselective deprotection (debenzoylation) of the obtained polymer and copolymer were carried out as described previously..$^{1,14,15}$

\section{Glycosylation}

Glycosylations with 2,3,4,6-tetra- $O$-benzyl$\beta$-D-glucopyranosyl trichloroacetimidate ${ }^{1}(\alpha-$ rich glucosylation) and 3,4,6-tri- $O$-acetyl- $\alpha$-Dglucopyranose-1,2-(tert-butyl orthoacetate) ${ }^{15}$ ( $\beta$-rich glucosylation) were performed as described previously.

\section{Debenzylation of Branched Polymers}

The branched polymer $(0.45 \mathrm{~g})$ dissolved in $10 \mathrm{ml}$ of dimethoxyethane was added dropwise to $50 \mathrm{ml}$ of liquid ammonia containing $0.6 \mathrm{~g}$ of sodium at $-78^{\circ} \mathrm{C}$. After $1.5 \mathrm{~h}$ ammonium chloride was added until the blue color completely disappeared, and then about $30 \mathrm{ml}$ of distilled water was added. After evaporation of ammonia, the debenzylated polymer suspension was washed several times with methylene chloride, and the solution was dialyzed for 3 days with water. The polymer was freeze-dried from water.

\section{Enzymatic Hydrolysis}

To the solution of $60 \mathrm{mg}$ of glucosylated polymer in $1 \mathrm{ml}$ of $0.05 \mathrm{M}$ acetate buffer $(\mathrm{pH}$ 4.8) was added $2 \mathrm{ml}$ of a buffered solution of cellulase ("Onozuka" 3S, Yakult Co., Ltd., Tokyo) ( $30 \mathrm{mg}, 90$ units). The reaction mixture was incubated at $45^{\circ} \mathrm{C}$ and the concentration of released glucose was measured by the glucose oxidase method. After $36 \mathrm{~h}$, the reaction was terminated by the addition of $1 \mathrm{ml}$ of ice-cold $50 \%$ trichloroacetic acid, and stored in an ice bath for $30 \mathrm{~min}$. The precipitated enzyme was removed by centrifugation and the supernatant was neutralyzed by addition of $0.5 \mathrm{~N} \mathrm{NaOH}$. After dialysis of polymer solution with water for 3 days, the polysaccharide was obtained by freeze-drying from the aqueous solution. 


\section{RESULTS AND DISCUSSION}

Control of Branching Distribution with Enzymatic Hydrolysis

Panaxan-like polysaccharide of $(1 \rightarrow 6)-\alpha-D-$ glucopyranan having $(1 \rightarrow 3)-\alpha$-D-glucopyranosyl branch was synthesized as described previously. ${ }^{1}$ Ring-opening polymerization of 1,6-anhydro-3- $O$-benzoyl-2,4-di- $O$-benzyl$\beta$-D-glucopyranose (1) with $\mathrm{PF}_{5}$ as catalyst at low temperature gave a highly stereoregular polymer, which was converted to 2,4-di- $O$ benzyl- $(1 \rightarrow 6)-\alpha$-D-glucopyranan by debenzoylation with sodium methoxide. The polymer was glucosylated according to the glycosyl imidate method ( $\alpha$-rich glucosylation $)^{1}$ and the orthoester method ( $\beta$-rich glucosylation $)^{15}$. Deprotection of the branched polysaccharide was carried out with sodium in liquid ammonia at $-78^{\circ} \mathrm{C}$ to give a $(1 \rightarrow 6)-\alpha$-D-glucopyranan having $\alpha$-D-glucopyranosyl and $\beta$-D-glucopyranosyl branches. Only the $\beta$-D-glucopyranosyl branch of the polymer was completely removed by enzymatic hydrolysis by the use of cellulase to provide stereoregular $(1 \rightarrow 6)$ - $\alpha$-D-glucopyranan having an $\alpha$-D-glucopyranosyl branch at the C-3 position as shown in Table I.

The branched polysaccharides having different anomeric ratios of the branching units ( $\alpha$-glucosyl branch : $\beta$-glucosyl branch), which were affected by glycosylation method and the glycosylation reaction condition, were treated with $\beta$-glucosidase (cellulase). In both $\alpha$ glucosyl-rich and $\beta$-glucosyl-rich branched polysaccharides, $\beta$-D-glucopyranosyl branches were completely removed by enzymatic hydrolysis using cellulase as confirmed by ${ }^{13} \mathrm{C}$ NMR spectroscopy (Figure 1). In each branched

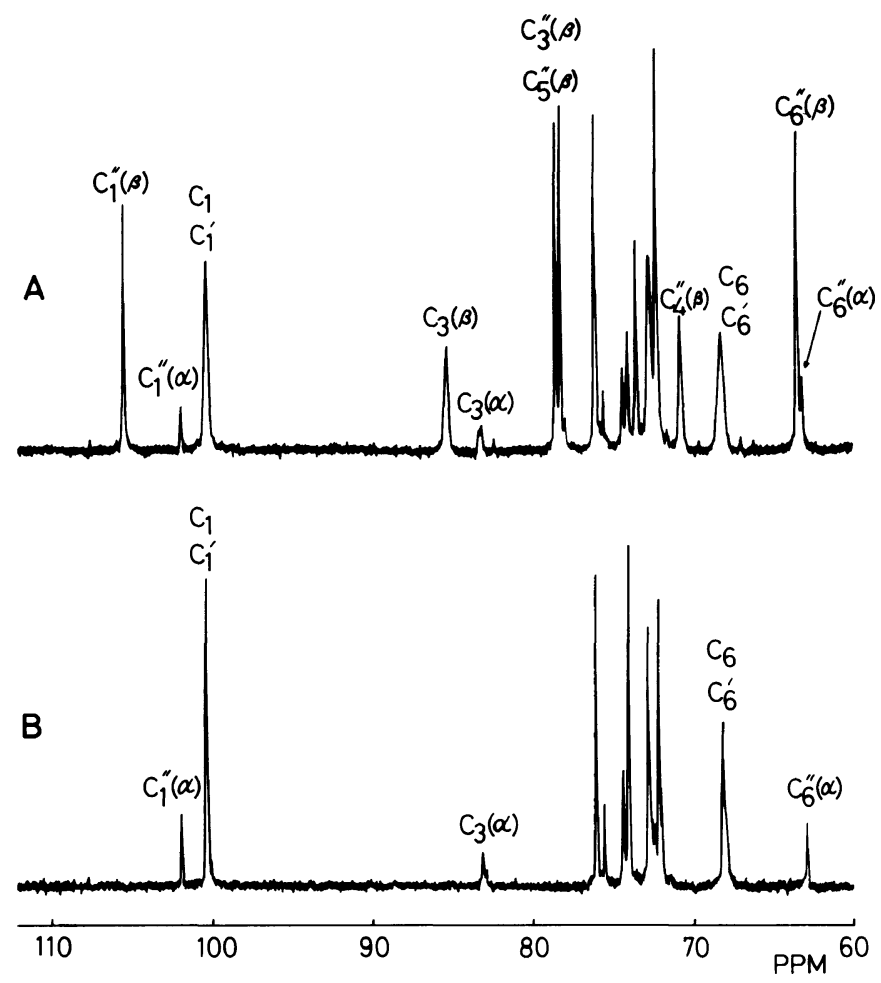

Figure 1. ${ }^{13} \mathrm{C}$ NMR spectra of (A) 3-O-( $\alpha, \beta$-glucopyranosyl-(1 $\left.\rightarrow 6\right)-\alpha$-D-glucopyranan and (B) cellulase treated polysaccharide. Peaks are assignable to carbons in three kinds of sugar residues as follows: $\mathrm{C}_{n}$ $(n=1-6)$, main chain unit with branching; $\mathrm{C}_{n}^{\prime}$, main chain unit without branching; $\mathrm{C}_{n}^{\prime \prime}$, branching sugar unit. 
Table I. Enzymatic hydrolysis of 3-O- $\alpha \beta$-D-glucopyranosyl-(1 $\rightarrow 6)-\alpha$-D-glucopyranan

\begin{tabular}{|c|c|c|c|c|c|}
\hline \multirow[b]{2}{*}{ Run no. } & \multicolumn{3}{|c|}{ Starting polymer } & \multicolumn{2}{|c|}{ Hydrolyzed polymer } \\
\hline & $\bar{M}_{n}^{\mathrm{a}}\left(\times 10^{4}\right)$ & $\begin{array}{l}\text { Content of } \\
\text { branch }^{\text {b }}\end{array}$ & $\begin{array}{l}\text { Anomeric ratio } \\
\text { of branching unit } \\
(\alpha: \beta)^{\mathrm{b}}\end{array}$ & $\bar{M}_{n}^{\mathrm{a}}\left(\times 10^{4}\right)$ & $\begin{array}{l}\text { Content of } \\
\text { branch }^{b}\end{array}$ \\
\hline 1 & 1.5 & 0.66 & $17: 83$ & 0.7 & 0.10 \\
\hline 2 & 1.9 & 0.52 & $75: 25$ & 1.8 & 0.38 \\
\hline 3 & 1.4 & 0.75 & $80: 20$ & 1.0 & 0.63 \\
\hline
\end{tabular}

a Determined by GPC.

${ }^{b}$ Culculated from ${ }^{13} \mathrm{C}$ NMR data.
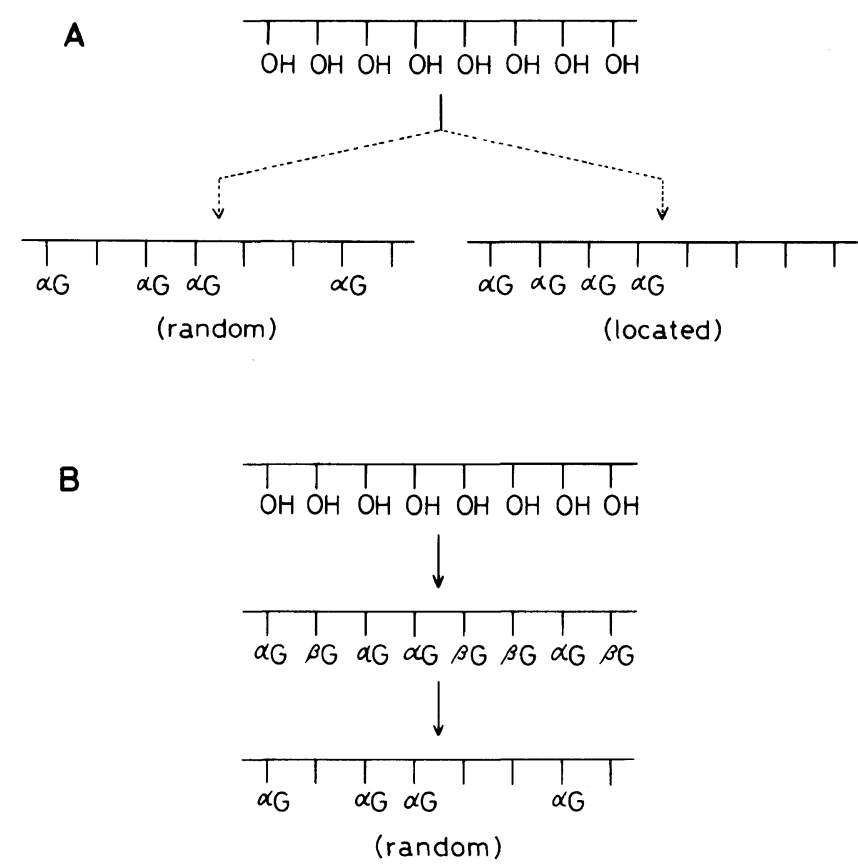

Figure 2. Branching distribution in $\alpha$-glucose-branched polysaccharide prepared by (A) stereoselective $\alpha$-glucosylation $(\alpha: \beta=100: 0, \mathrm{~d} s=x \%)$ and (B) nonselective glucosylation $(\alpha: \beta=x: 100-x, \mathrm{~d} s=100 \%)$.

polysaccharide, the content of $\alpha$-D-glucopyranosyl branch in the polysaccharide before enzymic reaction, which was calculated by branching content and anomeric ratio of the branch, was almost the same as the content of $\alpha$-D-glucopyranosyl branch in the polysaccharide after enzymic reaction, indicating that the $\alpha$-D-glucopyranosyl branch was hardly hydrolyzed by enzymic reaction. On the other hand, a comparison of molecular weights before and after the enzymatic hydrolysis suggested that main chain scission did not take place. Therefore, it was concluded that cellulase cleaved only $\beta$-glucosidic linkage and did not cleave $\alpha$-glucosidic linkage at all weather it is in main chain or in branch.

When the branching reaction on the linear polysaccharide is attempted, branching distribution may depend on the glycosylation method and glycosylation condition. For 
example, when the branched polysaccharide having $50 \%$ of $\alpha$-D-glucopyranosyl branch is the target compound, $50 \%$ glucosylation of the polysaccharide using stereoselective method $(\alpha: \beta=100: 0)$ can give the target branched polysaccharide, but the branching disribution (random, located, etc.) is uncontrollable (Figure 2A). Moreover, such branching distributions in the polysaccharide are difficult to be analyzed.

On the other hand, $100 \%$ glucosylation of the polysaccharide using nonselective glucosylation method (e.g., $\alpha: \beta=50: 50$ ) and subsequent enzymatic hydrolysis by cellulase may give the target polysaccharide with random branching (Figure 2B), since the anomeric selection $(\alpha$ or $\beta$ ) of the glucosylation reaction should be independent of the sequence, that is, both anomer branches should randomly distributed on the polymer.

In the case of our present experiment, branching contents were not $100 \%$ ( $52-75 \%$ as shown in Table I), indicating that the branching distributions of the obtained polysaccharides were not completely random.

\section{Copolymerization of $\mathbf{1}$ and $\mathbf{2}$}

In order to control the branching distribution further, the distribution of hydroxyl groups, which would be glycosylated, was attempted to be controlled by copolymerization reaction. Copolymerization of 1,6-anhydro-3- $O$-benzoyl-2,4-di- $O$-benzyl- $\beta$-D-glucopyranose (1) and 1,6-anhydro-2,3,4-tri- $O$ benzyl- $\beta$-D-glucopyranose (2) was carried out with phosphorus pentafluoride as catalyst in methylene chloride at low temperatures under high vacuum. The results are summarized in Table II. Copolymer composition, i.e., mole fraction of each monomeric unit in the copolymer was calculated by ${ }^{1} \mathrm{H}$ NMR spectroscopy, and stereoregularity of the copolysacharide was confirmed by ${ }^{13} \mathrm{C} \mathrm{NMR}$ spectroscopy.

As the mole fraction of $\mathbf{1}$ in the feed increased, polymer yield decreased and the specific rotation of the copolymer increased. The mole fraction of 1 unit in the copolymer was less than that of $\mathbf{1}$ in the feed, indicating that polymerizability of $\mathbf{1}$ was lower than that of 2 . The monomer reactivity ratios at $-60^{\circ} \mathrm{C}$ were evaluated by the Kelen-Tüdös method, ${ }^{16}$ which is applicable to data in relatively high conversions. The monomer reactivity ratios were $r_{1}=0.27$ and $r_{2}=2.5$.

The monomer composition in the feed which gives a certain composition of copolymer can be calculated by the monomer reactivity ratios. For example, mole fraction of 1 of 0.75 in the feed gives $1: 1$ copolymer. The calculated

Table II. Copolymerization of 1,6-anhydro-3- $O$-benzoyl-2,4-di- $O$-benzyl- $\beta$-D-glucopyranose (1) and 1,6-anhydro-2,3,4-tri- $O$-benzyl- $\beta$-D-glucopyranose $(2)^{\mathbf{a}}$

\begin{tabular}{|c|c|c|c|c|c|c|c|}
\hline \multirow{2}{*}{ No. } & \multirow{2}{*}{$\begin{array}{l}\text { Mole fraction } \\
\text { of } 1 \text { in feed }\end{array}$} & \multirow{2}{*}{$\frac{\text { Catalyst }}{\operatorname{mol} \%}$} & \multirow{2}{*}{$\frac{\text { Time }}{h}$} & \multirow{2}{*}{$\frac{\text { Yield }}{\%}$} & \multirow{2}{*}{$\begin{array}{l}\text { Mole fraction of } \\
\mathbf{1} \text { in copolymer }\end{array}$} & \multirow{2}{*}{$\frac{[\alpha]_{D}^{c}}{\operatorname{deg}}$} & \multirow{2}{*}{$\begin{array}{l}\bar{M}_{n}^{\mathrm{d}} \\
\times 10^{4}\end{array}$} \\
\hline & & & & & & & \\
\hline 1 & 0 & 1 & 0.5 & 27.9 & 0 & +110.3 & 28.0 \\
\hline 2 & 0.1 & 3 & 0.25 & 87.4 & 0.044 & +109.1 & 15.0 \\
\hline 3 & 0.3 & 5 & 0.33 & 43.5 & 0.124 & \pm 125.2 & 11.2 \\
\hline 4 & 0.5 & 10 & 1.0 & 29.4 & 0.265 & +125.6 & 6.1 \\
\hline 5 & 0.7 & 10 & 1.5 & 23.7 & 0.440 & +133.3 & 3.9 \\
\hline 6 & 0.9 & 10 & 1.5 & 7.0 & 0.715 & +149.3 & 3.2 \\
\hline 7 & 1.0 & 10 & 19.0 & 75.7 & 1 & +150.7 & 2.6 \\
\hline
\end{tabular}

a Monomer, $1.12 \mathrm{mmol}$; solvent, dichloromethane; catalyst $\mathrm{PF}_{5}$.

b Calculated from ${ }^{1} \mathrm{H}$ NMR data.

c Measured in chloroform $(c 1.0)$ at $25^{\circ} \mathrm{C}$.

d Determined by GPC. 


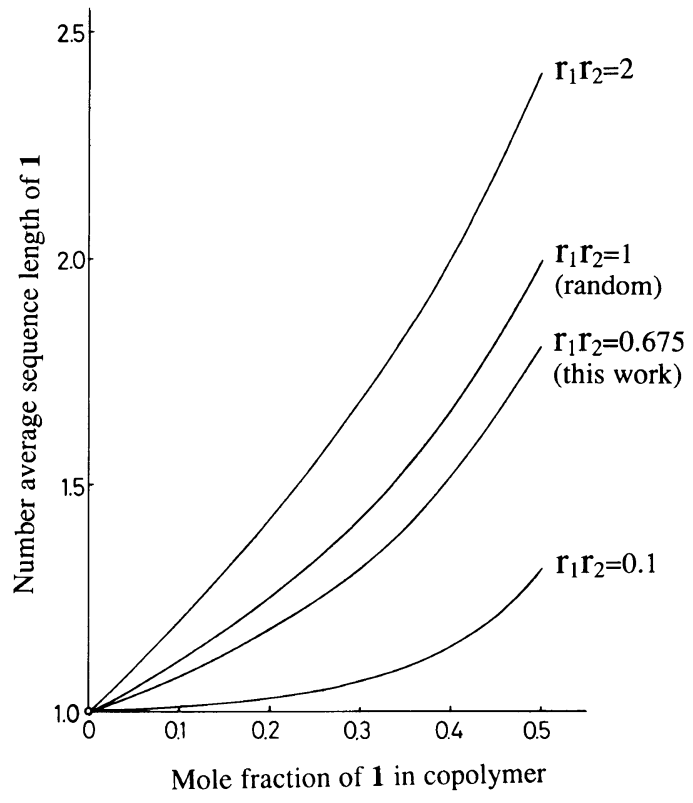

Figure 3. Effects of the copolymer composition and the product of the monomer reactivity ratios on the number average sequence length of each monomeric unit.

number average sequence length of each monomeric unit in the $1: 1$ copolymer was $1+\left(r_{1} r_{2}\right)^{1 / 2}$. Effects of the copolymer composition and the product of the monomer reactivity ratios on the number average sequence length were shown in Figure 3. The copolymerizations with different $r_{1} r_{2}$ value provides a certain composition of the copolymer with different sequence length. Therefore, the distribution of the hydroxyl groups (branching points) may be controlled by the product of the monomer reactivity ratios (kinds of monomers) and monomer composition in the feed. For example, the 1,6-anhydroglucose monomers with acetyl groups $\left(r_{1} r_{2}=0.198\right)^{17}$ or crotyl group $\left(r_{1} r_{2}=1.353\right)^{13}$ instead of benzoyl group $\left(r_{1} r_{2}=0.675\right.$, this paper $)$ as protective group at C-3 were copolymerized with 2 to give copolymers with a variety of monomeric distribution.

By a combination of the copolymeriza- tion and the enzymatic hydrolysis, the monosaccharide branch can be randomly introduced onto the sequencially controlled hydroxyl groups in polysaccharide main chain.

Acknowledgments. This work was supported in part by a Grant-in-Aid for Scientific Research (No. 04205040) from the Ministry of Education, Science, and Culture of Japan.

\section{REFERENCES}

1. K. Hatanaka, S. C. Song, A. Maruyama, T. Akaike, A. Kobayashi, and H. Kuzuhara, J. Carbohydr. Chem., 11, 1027 (1992).

2. A. Kameyama, H. Ishida, M. Kiso, and A. Hasegawa, J. Carbohydr. Chem., 10, 549 (1991).

3. S. I. Nishimura, K. Matsuoka, T. Furuike, S. Murayama, K. Nagami, S. Ishii, K. Kurita, and H. Kuzuhara, presented at the XVIth International Carbohydrate Symposium, Paris, 1992.

4. K. Hatanaka, Y. Ito, T. Akaike, K. Ishio, and T. Uryu, Glycoconjugate J., 8, 276 (1991).

5. M. Tomoda, K. Shimada, C. Konno, K. Sugiyama, and H. Hikino, Planta Med., 434 (1984).

6. M. Tomoda, K. Shimada, C. Konno, K. Sugiyama, and H. Hikino, Planta Med., 436 (1984).

7. E. R. Ruckel and C. Schuerch, J. Org. Chem., 31, 2233 (1966).

8. T. Uryu, H. Tachikawa, K. Ohaku, K. Terui, and K. Matsuzaki, Makromol. Chem., 178, 1929 (1977).

9. T. Uryu, K. Hatanaka, K. Matsuzaki, and H. Kuzuhara, Macromolecules, 16, 853 (1983).

10. B. Veruovic and C. Schuerch, Carbohydr. Res., 14, 199 (1970).

11. V. Masura and C. Schuerch, Carbohydr. Res., 15, 65 (1970).

12. K. Kobayashi, K. Nomura, and M. Okada, presented at the XVth International Carbohydrate Symposium, Yokohama, 1990.

13. H. Ito and C. Schuerch, J. Am. Chem. Soc., 101, 5797 (1979).

14. T. Uryu, M. Yamanaka, M. Henmi, K. Hatanaka, and K. Matsuzaki, Carbohydr. Res., 157, 157 (1986).

15. K. Hatanaka, T. Hirobe, T. Yoshida, M. Yamanaka, and T. Uryu, Polym. J., 22, 435 (1990).

16. T. Kelen and F. Tüdös, J. Macromol. Sci. Chem., A9, 1 (1975).

17. K. Kobayashi, H. Sumitomo, and A. Yasui, Polym. J., 14, 241 (1982). 